\title{
Der Augenöffner
}

Erhard Taverna

erhard.taverna[at]saez.ch
Gegen Ende seiner spezialärztlichen Ausbildung wollte Roman Graemiger, geboren 1961, sein Wissen und Können in der Dritten Welt erproben. So sei er, nach einiger Suche im Internet, auf die Augenklinik in Katmandu gestossen, wo er im Team des Chefarztes Dr. Sanduk Ruit neun Monate verbrachte. Zwei Jahre später übernahm er 1998 die Praxis seines Vaters in St. Gallen. 2003 wurde der Verein «VISION TIBET» gegründet, seit 2006 eine Stiftung, deren Vizepräsident er heute ist. Die Stiftung ergänzt als europäischer Partner die Projekte des nepalesischen Tilganga Institute of Ophthalmology TOI und des Himalayan Cataract Project HCP in Vermont, USA.

Das letzte Mal hat Roman Graemiger drei Wochen am Himalaya verbracht und in einem chirurgischen, mobilen Augen-Camp gearbeitet. Etwa alle zwei Monate werden diese Camps von Katmandu aus in abgelegenen Gegenden organisiert, um dort vor allem für Nomaden ohne Einkommen unentgeltliche Hilfe zu leisten. Zu den Zielen der Stiftung gehört die Finanzierung dieser temporären Einrichtungen, der Aufbau fester Augenabteilungen, zum Beispiel in Lhasa, Kailash und Xining, sowie die Ausbildung einheimischen Personals.

Nach längeren Fahrten in Lastwagen und Bussen, ausnahmsweise per Flugzeug, werden Schulhäuser zu Feldlazaretten umfunktioniert. Die einheimische Bevölkerung erfährt davon via Radio und Herolde, worauf sich bis zu tausend Menschen einfinden. Am ersten und zweiten Tag werden die Personalien aufgenommen, der Visus wird bestimmt und mit Hilfe von Spaltlampen eine Triage durchgeführt.

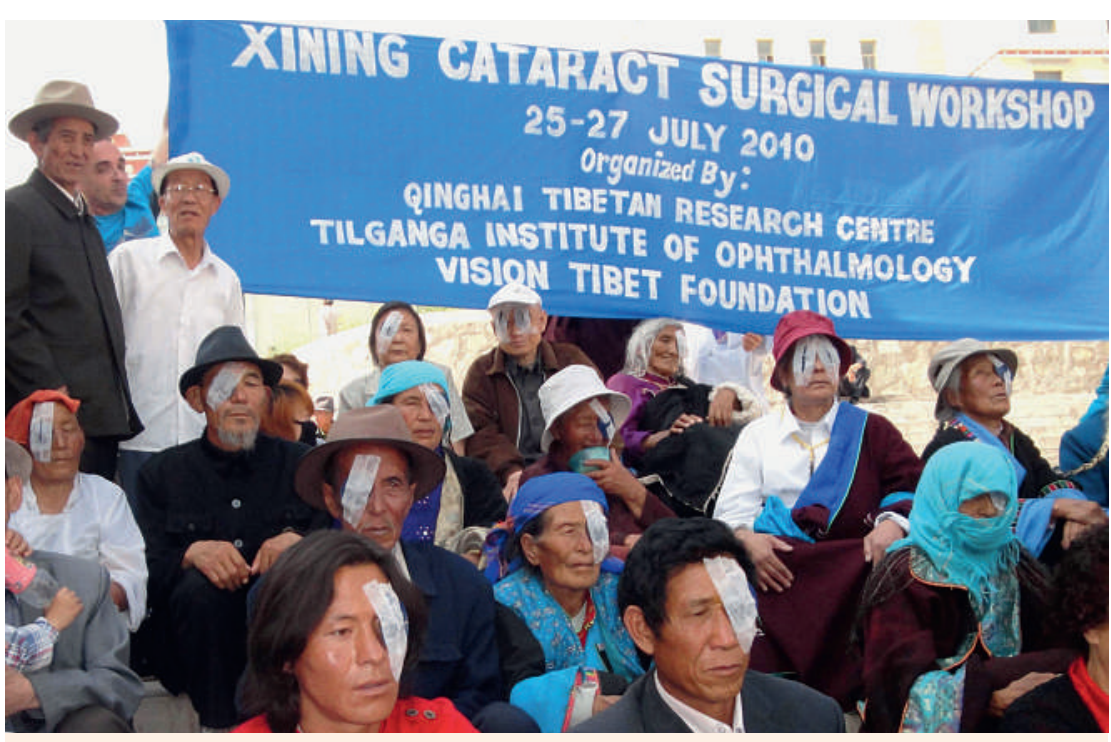

Behandlung des grauen Stars in einem Operationscamp in Xining, Region Amdo, Juli 2010, (c) Tibet Vision.
Rund ein Viertel der Untersuchten wird an den folgenden

Tagen operiert, der Rest erhält Medikamente oder ein einfaches Brillenrezept. Es kommen nur mobile und robuste Apparaturen zum Einsatz, Mikroskope mit einer Halterung an der Bahre oder Phako-Geräte der Firma Oertli aus Berneck. Gutvorbereitete Teams sorgen für die ganze Infrastruktur, für Autoklaven und Instrumente für die in Lokalanästhesie vorgenommenen Eingriffe. Am häufigsten sind dies Cataractoperationen, gefolgt von Lidkorrekturen, zum Beispiel nach einem Trachom, oder vereinzelt auch Schieloperationen bei Kindern. Zwei bis drei Ärzte sind an der Arbeit, einer davon steht für neu Hinzukommende oder Notfälle zur Verfügung. Nach dem ersten Verbandswechsel und einer Instruktion gehen die Leute wieder nach Hause. Vier bis sechs Wochen später erfolgt an Ort, sofern nötig, eine nichtärztliche Nachkontrolle. Der graue Star ist das Hauptübel der Bergbevölkerung. Die Ursachen sind nicht völlig geklärt, genetische Faktoren, Mangelernährung, UVStrahlen und auch der Rauch offener Feuerstellen sind vielleicht für die weltweit grösste Rate von Linsentrübungen verantwortlich. Fast jeder fünfte Tibeter über vierzig Jahre ist davon betroffen. Implantiert wird eine intraokulare Linse, die dank der australischen Fred Hollows-Foundation in perfekter Qualität in Katmandu produziert und auch nach Indien, Pakistan und China exportiert wird. Das gleichwertige Produkt der einheimischen Techniker kostet rund 10 Franken (bei uns sind es 500-1000 Franken).

Inzwischen gibt es weitere Kliniken in Lhasa, Tingri und Xining, die ihrerseits mit mobilen Equipen dazu beitragen, dass jährlich 3000-5000 Patienten operiert und weit über 1000 Konsultationen geleistet werden. Trotz dieser Erfolge bleibt der graue Star ein Problem, da die Bevölkerung im Himalaya zunimmt und älter wird. Roman Graemiger plant bereits seine nächste Reise von der Dauer einer schweizerischen WK-Länge. Bisher als Solist, was er bedauert, denn die Arbeit bereichere ihn menschlich und beruflich. Er ist gerne bereit, interessierten Kollegen oder Kolleginnen seiner Fakultät die nötigen Auskünfte zu erteilen. Wer das Unternehmen von VISION TIBET passiv unterstützen möchte oder an Vorträgen und einem Dokumentarfilm über ein Operationsteam im Westtibet interessiert ist, bekommt weitere Informationen unter www.visiontibet.ch.

Kontakt: Dr. Roman Graemiger, Bahnhofplatz 1, 9001 St. Gallen, Tel. 0712228866 